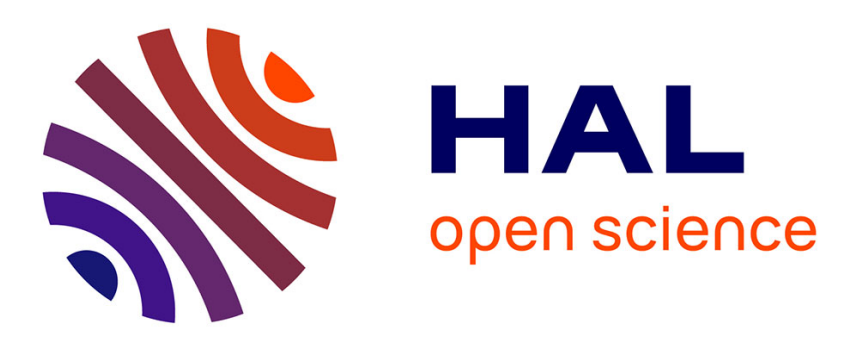

\title{
La nation face à l'État et aux rapports de classe : quels enjeux contemporains pour la politique économique ?
}

Jérôme Maucourant, Bruno Tinel

\section{To cite this version:}

Jérôme Maucourant, Bruno Tinel. La nation face à l'État et aux rapports de classe : quels enjeux contemporains pour la politique économique ?. Actuel Marx, 2020, 2020/2 (68), pp.108-124. 10.3917/amx.068.0108 . halshs-02955449

\section{HAL Id: halshs-02955449 \\ https://shs.hal.science/halshs-02955449}

Submitted on 1 Oct 2020

HAL is a multi-disciplinary open access archive for the deposit and dissemination of scientific research documents, whether they are published or not. The documents may come from teaching and research institutions in France or abroad, or from public or private research centers.
L'archive ouverte pluridisciplinaire HAL, est destinée au dépôt et à la diffusion de documents scientifiques de niveau recherche, publiés ou non, émanant des établissements d'enseignement et de recherche français ou étrangers, des laboratoires publics ou privés. 


\title{
La Nation face à l'Etat et aux rapports de classe, quels enjeux contemporains pour la politique économique?
}

Jérôme Maucourant

Université Jean-Monnet de Saint-Etienne et Triangle, UMR 5206

maucourant.jerome@,wanadoo.fr

Bruno Tinel

Université Paris 1 Panthéon-Sorbonne et Centre d'Economie de la Sorbonne, UMR 8174.

bruno.tinel@univ-paris1.fr

\section{Résumé}

Comment caractériser les contradictions entre l'Etat et la nation, tout en prenant en compte le fait que la société réelle est divisée en classes ? Ces éléments sont abordés sous l'angle des implications d'une politique de plein emploi, à partir de l'analyse de Kalecki. Puis, l'idée d'une double nature de l'Etat, dont on trouve des embryons chez Marx, est mobilisée pour articuler les notions d'Etat, de nation et de classes sociales. Enfin on s'interroge sur les enjeux actuels de la politique économique face aux tendances qui sont censées éroder le rôle de l'Etat et des nations.

\begin{abstract}
How can we characterize the contradictions between the state and the nation, while taking into account the fact that real society is divided into classes? This paper analyses these elements based on the implications of a full employment policy following Kalecki's analysis. We also find that to articulate the notions of state, nation and social classes it is relevant to examine the idea of a dual nature of the state, of which certain elements are found in Marx. Finally, we address the current challenges of economic policy taking into account the trends that tend to erode the role of the state and nations.
\end{abstract}

Dans nos mondes contemporains, la politique économique correspond aux choix effectués par le gouvernement, c'est-à-dire le sommet de l'Etat, pour agir sur la trajectoire de l'économie nationale. Comment caractériser les contradictions à l'œuvre entre l'Etat et la nation, dès lors que l'on prend en compte le fait que la société réelle, dont il est question sur un espace national donné, est divisée en classes ? La présente contribution tente d'articuler les faits sociaux actuels que sont la nation, l'Etat et les rapports de classes en prenant la politique économique comme point de départ. Plus particulièrement, le fait que les gouvernements aient la possibilité matérielle de réaliser le plein emploi, sans pour autant y recourir, demeure aujourd'hui trop peu discuté du point de vue de l'économie politique. Quels sont les effets d'une telle politique économique sur les rapports de classe et comment ces mêmes classes sont-elles conduites à réagir ? Examiner ces trois éléments combinés (Etat, nation, rapports de classes) permet de caractériser des contradictions structurelles entre ces termes. Sans prétendre que celles-ci soient indépassables, il s'agit au moins d'admettre qu'elles sont certainement amenées à durer longtemps. A l'échelle de nos vies, et même des générations à venir, elles ont très probablement un caractère permanent, dont ceux qui prétendent vouloir agir sur la réalité sociale, plutôt que simplement la contempler, devraient davantage tenir compte.

Alors que les approches s'inscrivant dans la démarche de Marx soulignent presque immanquablement, et en premier lieu, que l'Etat est au service des classes dominantes, ce qui est peu contestable, il serait un peu court d'en rester là ${ }^{1}$. Depuis Marx, en effet, l'histoire a vu surgir des faits nouveaux de premier ordre qui sont, trop souvent, considérés comme négligeables dans la

\footnotetext{
${ }^{1}$ Pour une introduction à la théorie de l'Etat chez Marx, cf. Herrera, Rémy, Brève introduction à la théorie de l'État chez Marx et Engels, Cahiers de la MSE, série Rouge, R01001, $2001<\mathrm{ftp} / / \mathrm{mse}$.univparis1.fr/pub/mse/cahiers2001/R01001.pdf>
} 
littérature critique ${ }^{2}$. On peut en mentionner tout particulièrement deux : 1/ l'émergence de la politique économique dans les années 1930, avec la capacité pour l'Etat de modifier la trajectoire d'accumulation, au point de lui permettre d'atteindre le plein emploi, et 2/ l'émergence de l'Etat social (welfare state), lequel est constitué d'un ensemble de dispositifs assurantiels collectifs et contraignants conduisant à soustraire très largement la reproduction de la force de travail à la loi de la valeur. Ainsi, nos sociétés modernes en régime capitaliste ont été le théâtre d'une socialisation du revenu à des niveaux ${ }^{3}$ que ni Marx et Engels, ni Lénine, n'avaient jamais imaginés autrement que sous les termes du socialisme. Mais il y a plus : ce socialisme, incomplet et imparfait, qui a émergé dans et par des institutions politiques, à la fois bourgeoises et imparfaitement démocratiques, a vu le jour et continue d'exister, y compris en Europe, à l'échelle des nations. Les rapports contradictoires entre Etat, nation et classes sociales doivent être interprétés de manière à tenir compte de ces faits majeurs dans leur déploiement historique, sous peine d'enfermer le marxisme dans une gnose.

\section{Les enjeux de classe d'une politique de plein emploi dans une économie capitaliste}

Dans un court article assez connu, datant 1943, Michal Kalecki qui fut l'un des premiers marxistes à penser la politique économique ${ }^{4}$ écrivait en substance que si les problèmes économiques pour parvenir au plein emploi ${ }^{5}$ étaient surmontables, grâce à une politique budgétaire volontariste, en revanche le plein emploi faisait naître des problèmes politiques autrement plus épineux. Il note en particulier la réticence violente des classes financières et industrielles face au new deal aux ÉtatsUnis, face au gouvernement Blum en France et dans l'Allemagne pré-hitlérienne, à s'orienter vers

${ }^{2}$ Sans prétendre être exhaustifs, deux exceptions notables dans le monde francophone doivent être mentionnées ici : Friot, Bernard, Puissances du salariat, Paris, La Dispute, (1998) 2012 et Hoang-Ngoc, Liêm, Refermons la parenthèse libérale, La Dispute, 2005. Le fait que ces deux ouvrages soient publiés par La Dispute n'est cependant pas un hasard.

${ }^{3}$ Par exemple, en France, les prestations sociales atteignent un tiers du PIB. Plus on descend dans la distribution des revenus, plus la part des prestations sociales est grande dans le revenu disponible des ménages : « en 2017, les prestations sociales représentent $46 \%$ du revenu disponible des $10 \%$ d'individus les moins aisés » (cf. Insee Première, $\mathrm{n}^{\circ} 1772$, sept. 2019). Si l'on regarde le revenu des ménages au sens large (le revenu disponible ajusté brut, RDAB, qui prend en compte les prestations en espèces et les transferts sociaux en nature, c'est-à-dire les dépenses socialisées d'éducation et de santé), c'est désormais $50 \%$ du RDAB agrégé des ménages qui est socialisé (cf. Insee, comptes de la nation). Certes la France fait partie des pays les plus avancés en la matière mais les autres économies capitalistes ont connu des trajectoires tout à fait similaires ; aujourd'hui, un milliard d'êtres humains sont concernés par ces mécanismes de socialisation massive à l'échelle des nations.

4 CF. Kalecki, Michal, Political Aspects of Full Employment, Political Quarterly, 1943, 14(4): $322-31$ (https://delong.typepad.com/kalecki43.pdf). Cet économiste marxiste polonais (1899-1970) est l'un des fondateurs, avant la lettre, de ce qu'on appellera après-guerre le keynésianisme. Les modèles post-keynésiens contemporains sont essentiellement des modèles kaleckiens, ils prennent notamment en compte le fait que les nations sont divisées en classes aux intérêts conflictuels, si bien que la répartition salaires-profits, centrale chez Ricardo et Marx, se retrouve également au cœur de ses analyses. Il s'intéresse aux conditions macroéconomiques qui rendent possibles l'extorsion de la plus-value, c'est-à-dire la réalisation des profits. Alors que, pour Luxemburg et Lénine, la demande induite ne peut solvabiliser l'offre dans une économie capitaliste que par l'expansion coloniale, car les salaires sont par définition inférieurs à la valeur produite, Kalecki montre (à peu près en même temps que Keynes) qu'il n'en est rien pourvu qu'existe un élément autonome de la demande globale, ce qui est le cas de l'investissement. Dès lors, en économie fermée, c'est la dépense autonome d'investissement qui détermine les profits, et le niveau d'emploi pour un salaire et une propension à consommer donnés. Pour une vue d'ensemble concernant la pensée de Kalecki sur l'État et le capitalisme, cf. Kriesler, P. and B. McFarlane, Michal Kalecki on capitalism, Cambridge Journal of Economics, 1993, $17: 215-234$.

5 Comme on peut l'imaginer la notion de plein emploi fait l'objet de débats dont il n'est pas possible de rendre compte ici faute de place. En résumé, deux pôles s'opposent : pour les uns l'économie se trouve au plein emploi lorsque seule une infime minorité de travailleurs se retrouve au chômage de manière très temporaire, le temps de passer d'un emploi à un autre, pour les autres le plein emploi correspond au niveau d'emploi qui n'accélère pas l'inflation dans ce cas il existerait un taux de chômage optimal, associé à un taux d'inflation constant. Malgré son imprécision, le présent article retient la première définition. 
le plein emploi durant les années $1930^{6}$. Le plein emploi correspond à un niveau élevé de l'activité, il ne bénéficie donc pas uniquement aux travailleurs puisque les capitalistes réalisent un volume maximal de profits. Pourquoi dès lors, interroge Kalecki, les classes possédantes refuseraient-elles les promesses d'un boom et sa moisson de profits considérables ? Ce d'autant plus que, pour y parvenir, il n'est pas question d'accroître les impôts sur le capital, mais la dépense et les dettes publiques, lesquelles servent d'outil de placement pour l'épargne des classes fortunées.

Kalecki souligne que, si le gouvernement peut agir directement par sa dépense sur le niveau de l'emploi, alors le monde des affaires, en tant que communauté, perd de sa capacité à influencer la politique économique. C'est-à-dire qu'en la matière, le gouvernement peut possiblement s'élever audessus des rapports de classes. À l'opposé, dans un système où règne une pratique de laisser-faire, comme c'est le cas dans nos économies depuis l'abandon des politiques keynésiennes au début des années 1980, le gouvernement ne cherche pas à fixer le niveau de l'emploi, il en laisse le soin au " marché », c'est-à-dire en fait aux choix agrégés des capitalistes. Dans ce monde dit " libéral », le niveau d'emploi dépend de «l'état de la confiance » des entrepreneurs : il ne faut rien faire qui puisse heurter la communauté industrielle et financière sinon la confiance se dégrade et, avec elle, l'activité et l'emploi plongent également. Le monde du laisser-faire est un monde où l'État est aux « petits soins » pour les « capitaines d'industrie » : pour le Bien public, il faut qu'ils se sentent à leur aise avec la politique du gouvernement. Néanmoins, dès lors que le gouvernement peut agir directement sur l'activité par sa dépense autonome, l'influence des dirigeants économiques diminue, c'est-à-dire qu'ils voient s'émousser leur capacité à exercer un chantage à l'emploi. Kalecki souligne que les discours sur l'orthodoxie budgétaire servent précisément à restaurer la capacité du monde des affaires à fixer, en tant que classe, le niveau de l'emploi et à influer sur la politique économique. Autre élément, le plus fondamental selon Kalecki pour expliquer l'opposition patronale au plein emploi : lorsque le chômage est faible, il est facile pour les salariés de trouver un nouvel emploi si bien que la menace de licenciement n'est plus effective pour discipliner la main d'œuvre, celle-ci devient incontrôlable tant à l'intérieur de l'entreprise qu'à l'extérieur. Au plein emploi, écrit Kalecki, les patrons redoutent que la conscience de classe des travailleurs ne soit affermie, ce qui les conduirait à multiplier les grèves pour obtenir des hausses de salaires et des améliorations dans leurs conditions de travail. Anticipant la boucle prix-salaires qui s'est formée dans les années 1970, il ajoute que les « capitaines d'industries » redoutent que les hausses de salaires ne provoquent une inflation destructrice pour le capital rentier.

Le plein emploi est un facteur de subversion des rapports de classe. Selon les alliances de classes qui se nouent pour exercer le pouvoir politique, l'État va, ou non, laisser (par la dynamique de la dépense publique) la détermination du niveau de l'emploi entre les mains des capitalistes. Instinctivement, les classes industrielles et financières considèrent le chômage comme faisant partie

6 En Allemagne, écrit Kalecki, l'attitude des classes économiques dirigeantes à l'endroit du plein emploi changea avec l'avènement du régime nazi. Pour l'auteur, l'une des fonctions du fascisme, que l'on retrouve dans le nazisme, était de lever les objections capitalistes face au plein emploi car l'État passe alors sous le contrôle partagé du big business et du fascisme, ce dernier s'occupe de faire régner l'ordre par la force. Toutefois, note l'auteur, comme ces régimes ont la particularité de privilégier une dépense publique centrée sur l'armement, ce qui les transforme en superpuissances militaires, ils marchent inévitablement vers la guerre. Ce genre de considération est peu à peu occulté, à mesure que l'on s'éloigne des conditions concrètes d'émergence des fascismes. Ainsi, Karl Polanyi, contemporain des événements, produit une analyse assez similaire et met en évidence la contradiction entre démocratie (politique) et économie (capitaliste) qui peut, dans certaines circonstances, déboucher sur le fascisme (cf. Polanyi, Karl, Le marxisme redéfini, 1934, dans Cangiani, Michele et Maucourant, Jérôme dir., Essais de Karl Polanyi, Seuil, 2008, p. 431-6). Celui-ci se caractérise par l'extirpation des éléments de démocratie existant dans les institutions de façon à sauver l'ordre fondé sur la propriété privée. Cela rappelle au passage que, tout contradictoire qu'il soit, le « capitalisme démocratique » n'est pas identique au fascisme comme le prétend au même moment une certaine gauche moquée par Orwell, cette gauche qui n'eut de cesse de fuir l'Allemagne dès que le fascisme réel prit le pouvoir (Orwell, George, Fascisme \& démocratie, 1941, dans Écrits politiques (1928-1949) - Sur le socialisme, les intellectuels \& la démocratie, Agone, Marseille, 2009, p. 168). Forme concrète de « capitalisme démocratique », l'Etat social n'est décidément pas une illusion. 
du régime capitaliste «normal ». Maintenir le contrôle, par le sous-emploi, à la fois sur la production et sur la vie politique est plus important qu'une flambée, jugée instable, des profits à l'occasion du plein emploi ${ }^{7}$.

\section{Les fondements de classe de la politique anti-keynésienne}

Révélé par le New Deal et théorisé par Kalecki et Keynes, le pouvoir potentiel de l'État dans une « démocratie capitaliste » sur la dynamique du capital et des rapports de classes est considérable. En défense de leurs intérêts de classe, les cercles économiques dirigeants, explique Kalecki, exigent que l'État agisse uniquement face aux récessions et s'abstienne d'assurer un plein emploi permanent. Cette exigence patronale rappelle étrangement les postulats théoriques qui s'imposeront quarante ans plus tard dans la macroéconomie dominante (dite néo-keynésienne) des années 1980 et 1990 : accepter des politiques de stabilisation passives mais postuler qu'elles n'ont pas d'effet à long terme, et donc « laisser faire » les agents privés pour déterminer le niveau de l'emploi. Le gouvernement peut être keynésien à court terme, mais pas trop quand même, et il doit être classique à long terme.

D'autre part, nous dit Kalecki, l'intervention publique est tolérée par les dirigeants industriels et financiers si elle vise à stimuler l'investissement privé par des baisses de taux d'intérêts, des réductions d'impôts et des subventions au secteur privé, et non pas à investir directement dans l'économie par le budget public et la socialisation de l'investissement. Ici encore, on ne peut être que frappé de voir combien ces exigences politiques du patronat, que Kalecki identifie très tôt face à l'essor du keynésianisme, finiront par constituer les bases de la macroéconomie orthodoxe antikeynésienne ${ }^{8}$ quarante ans plus tard. Ce qui était perçu par Kalecki en 1943 comme une revendication de classe se verra promu au rang de vérité scientifique par la seconde synthèse orthodoxe à partir des années 1980 .

Kalecki se montre lucide et visionnaire, à tel point que, 75 ans plus tard, il est encore intéressant de lire ce petit texte. Mais il va plus loin. Il considère en effet, à juste titre, que ces stimulations (ces « incitations » dit-on dans la science économique contemporaine) de l'investissement privé permettent certes d'amortir un peu l'ampleur du cycle mais sont beaucoup moins efficaces que l'investissement public si bien que s'installe un niveau d'emploi bien inférieur à ce qu'il pourrait être en cas d'action directe par l'État. Surtout note-t-il, ces mesures « incitatives » n'ont qu'un effet temporaire si bien que se profile bientôt un nouveau ralentissement qui doit alors pousser les autorités à réduire à nouveau le taux d'intérêt et les impôts sur le capital. Bientôt, il devient nécessaire de réduire de façon permanente le taux d'intérêt et l'impôt des capitalistes à tel point que le taux d'intérêt devient négatif, tout comme l'impôt qui se mue alors en subsides permanents versés aux entreprises.

Dans ces quelques lignes, Kalecki entrevoit ce que sera la « grande modération » des années 19902000 et leur dégénérescence en grande stagnation durant les années 2010 avec des taux d'intérêts nominaux passant sous la barre du zéro et une multiplication de la dépense fiscale à destination des entreprises, au point d'en arriver à les subventionner par des dispositifs divers, comme par exemple le CICE, d'abord temporaire puis pérennisé. Les grandes lignes des enchaînements macroéconomiques, qui se sont produits au cours des quatre dernières décennies dans l'ensemble des pays capitalistes avancés ayant adopté des politiques anti-keynésiennes, sont anticipés et décrits

7 La macroéconomie anti-keynésienne, qui présidera à celle du nouveau consensus dans les années 1980, se bâtira dans les années 1970 sur l'idée qu'il existerait un niveau de chômage jugé « naturel ». Cette naturalisation d'un rapport social lui confère un ancrage idéologique relativement indépassable. En d'autres termes, une autre politique économique ne peut pas être bâtie sur la théorie et les modèles du nouveau consensus.

8 Pour des exposés critiques, cf. Creel, Jérôme, Bruno Ducoudré, Catherine Mathieu et Henri Sterdyniak (2005) Doiton oublier la politique budgétaire ? Une analyse critique de la nouvelle théorie anti-keynésienne des finances publiques, Revue de l'OFCE, 2005, n 92 : 43-97 ; Arestis, Philip, Economic Policies of the New Consensus Macroeconomics: A Critical Appraisal, in Jocelyn Pixley and G. C. Harcourt (ed.) Financial Crises and the Nature of Capitalist Money, Palgrave Macmillan, 2013: 196-215. 
très simplement dans cet article. Bien que Kalecki ne le signale pas, on peut remarquer que les baisses d'impôts répétées en faveur des capitalistes et le faible dynamisme de l'activité, qui résulte de cette politique, conduisent à une dégradation permanente des comptes publics, et donc à une hausse continuelle des ratios de dettes publiques ${ }^{9}$, ceci est une autre caractéristique du régime d'accumulation actuel.

Enfin, avec prémonition, l'auteur considère que se formera une alliance du big business et de la petite propriété rentière pour exiger des finances publiques équilibrées, c'est-à-dire refuser le plein emploi permanent. Mais si l'économie ne reste pas au plein emploi, ceci signifie que les récessions obligent l'État à intervenir à intervalles réguliers pour relancer l'activité à la demande des « capitaines d'industries », si bien que ce n'est qu'en phase haute de cycle que l'économie se rapproche du plein emploi. Dans cette configuration, les cycles sont plus courts et de moindre ampleur. Au final, les cercles possédants et dirigeants peuvent se satisfaire d'un keynésianisme temporaire et très modéré : il permet d'éviter le pire pour les affaires tout en maintenant les salariés en situation de faible capacité de négociation.

\section{La constitutionnalisation de la politique économique contre les nations}

Au début des années 1980, le consensus autour de ces principes a été obtenu dans l'ensemble des pays capitalistes avancés par les classes dominantes au sein d'une bonne partie des dirigeants politiques toutes tendances confondues ${ }^{10}$. Cette réaction anti-keynésienne fut telle que les dogmes $\mathrm{du}$ « nouveau consensus » furent constitutionnalisés sous forme d'injonctions relatives à la lutte contre l'inflation et contre les déficits publics. C'est ainsi que la politique économique a été volontairement dépolitisée, c'est-à-dire soustraite des processus démocratiques. Les institutions européennes sont conçues de manière à limiter le champ des décisions prises par les gouvernement élus, au profit d'agences non contrôlées par le corps politique et d'un pilotage technocratique centré sur des règles d'applications présentées comme quasi-automatiques. En France, dont les dirigeants politiques n'ont pas été en reste dans cette conversion de masse au rocardo-balladurisme, tout ceci fut fait non pas directement à l'initiative du patronat et des représentants politiques du capital mais sous l'impulsion d'un parti qui se réclama, jadis, des idéaux du socialisme. Ceci n'a bien évidemment pas aidé les classes salariées à maintenir la conscience de leurs intérêts, et ce fut difficile pour elles de se mobiliser pour les défendre.

Depuis quarante ans, les gouvernements ont agi à l'encontre des intérêts des travailleurs et des citoyens avec un systématisme tellement stupéfiant et tellement biaisé en faveur des possédants fortunés, qu'on peut s'étonner de la patience de nos concitoyens et de la lenteur de la décomposition/recomposition politique (finalement entamée dans de nombreux pays au cours de la décennie 2010) ${ }^{11}$. Ce sont, paradoxalement, les acquis de la consommation de masse et la puissance de l'Etat social qui ont engendré des tendances individualistes et hédonistes, ce qui a entamé l'efficacité des actions collectives. D'ailleurs, les lamentations sur la « tentation populiste » font sourire. N'est-ce pas un juste retour des choses ? Cette « tentation populiste » n'est-elle pas la forme première de la contestation populaire après la liquidation des structures syndicales et politiques du salariat, liquidation qui fut tant souhaitée par les classes dominantes?

\section{La double nature de l'Etat}

La lecture de Kalecki a mis en évidence le fait que, dans une économie dominée par les rapports capitalistes, l'État a depuis près d'un siècle les moyens matériels de mener une politique

9 Cf. Tinel, Bruno, Dette publique sortir du catastrophisme, Raisons d'Agir, 2016.

${ }^{10}$ Plus personne ne se réclamait du keynésianisme puisque, depuis les années 1930, les organisations politiques se pensant comme « révolutionnaires » ont considéré le new deal et le keynésianisme comme une ruse de la raison bourgeoise pour détourner les masses prolétariennes de la vraie révolution.

${ }^{11}$ Sur ces questions, voir, Renault, Emmanuel, Tinel, Bruno, Les crises du néolibéralisme : processus de révoltes et adaptation. Actuel Marx, 2010, 47(1) : 100-117. 
économique qui passe en quelque sorte « au-dessus » des rapports de classes. Il l'a effectivement montré avec le New Deal et les politiques sociales démocrates menées en Europe après-guerre. L'État peut mener une politique économique de plein emploi et de promotion de l'État social, qui améliore objectivement le bien-être du plus grand nombre, contre la volonté des classes dominantes mais sans remettre en questions l'ordre de classe. Dans les systèmes économiques modernes, où règne -en partie- la marchandise et l'appropriation privée, l'État doit donc être pensé comme une entité contradictoire, ou en tension entre deux impératifs ou deux pôles. Dans les limites spatiales de son territoire, cette entité est, d'une part, conservatrice de la structure de classe et, d'autre part, dépositaire de l'intérêt national, lequel s'identifie à un au-delà des rapports classes. L'État est donc à la fois État de classe et État-nation ${ }^{12}$.

Dans les sociétés de classes, l'État est par définition un État de classe : c'est l'entité qui permet la reproduction rationalisée de la structure de classe, c'est-à-dire l'ordre social, par le biais d'une administration. Dans ce sens, il contribue à la reproduction d'ensemble de la société dans ses aspect matériels, intellectuels et culturels. Par l'État transitent, en dernier ressort, les dispositifs contraignant tous ceux qui ne font pas partie de l'élite dirigeante ou des classes dominantes à rester dans une relation de subordination de classe, ce qui n'empêche pas des individus de passer d'une classe à une autre. Avec l'essor et la rationalisation de l'Etat par le droit, une armée permanente, une police, une administration fiscale etc., les classes dominantes en viennent à leur tour à être subordonnées à l'Etat et ses procédures objectivées. C'est cette seule instance qui garantit leurs privilèges et est en mesure de reproduire leurs droits d'accès aux ressources et aux honneurs. Par nature, l'État est du côté des classes dominantes puisque, sans lui, elles seraient submergées et détruites par le nombre mais, bien avant les révolutions anglaise et française, il tend à intégrer les classes dominantes dans sa logique propre ${ }^{13}$. Un type d'État est intrinsèquement lié à une structure de classe donnée, les deux éléments coévoluent au fil des luttes sociales et des crises. Cette thèse exprime une position largement partagée au sein du marxisme, elle a été en quelque sorte codifiée par Lénine dans L'État et la Révolution, puis reprise et perfectionnée par Poulantzas qui considère l'État comme l'arbitre des contradictions entre les fractions des classes dominantes, ce qui le rendrait relativement autonome vis-à-vis de celles-ci ${ }^{14}$.

Cette idée, fondamentale, d'un État indispensable aux classes dominantes pour reproduire leur position de classe est insupportable à l'individualisme bourgeois qui préfère l'idéologie ${ }^{15}$ à la réalité. Dans la France de l'Ancien Régime, la noblesse se considérait elle-même comme partie intégrante de l'État monarchique. L'État était alors ouvertement la chose des dominants : aristocratie guerrière devenue foncière, d'abord, puis « noblesse de robe ». Ces privilégiés ne paient pas l'impôt par principe. La double réalité de sa domination et de son incarnation dans l'État n'était, sur le plan

${ }^{12}$ Loin des poncifs simplistes, on retrouve cette dualité dans l'analyse, en 1843, de l'Etat bourgeois par Marx entre la démocratie « vraie » et l' « Etat médiéval », cf. « La contradiction de l'état et la mutation de ses formes » dans Desbrousses, Hélène, L'apport de Marx à la théorie de l'Etat, Nouvelles Fondations, 5 (1) : 71-84, https://www.cairn.info/revue-nouvelles-fondations-2007-1-page-71.htm

${ }^{13}$ Cette idée est exposée par Norbert Elias lorsqu'il explique que la concurrence pour le double monopole fiscal et militaire fait place à une concurrence dans ce double monopole, constitué en administration, à mesure que s'affirme une autorité centrale au sein de l'aristocratie guerrière, cf. Elias, Norbert La dynamique de l'Occident, Pocket, (1939) 2003.

${ }^{14}$ En réalité, « l'autonomie » de l'Etat est sans doute intrinsèque au concept d'Etat lui-même : soit l'Etat est la classe dominante, et il n'est pas autonome (l'« Etat médiéval » qu'évoque Marx en 1843), soit il est distinct de la classe dominante, et il est donc autre chose, une chose pourvue d'une autonomie, nécessairement relative (connaît-on une seule chose dans l'univers social, caractérisé par l'interdépendance, qui soit totalement autonome du reste ?). Dans ce cas, disserter sur « l'autonomie relative de l'Etat» reviendrait à s'étendre sur une redondance.

15 En voici le contenu : les classes dominantes se suffisent à elles-mêmes, d'ailleurs ce ne sont pas des classes mais uniquement des individus méritants, capables d'épargne, de travail et d'innovation, interagissant librement sur le marché, tandis que l'État serait là uniquement pour promouvoir le bien public. Sur l'idéologie, nous utilisons ce terme dans le sens de Marx, tel que Patrick Tort l'a formalisé, cf. Tort, Patrick, Marx et le problème de l'idéologie, PUF, 1988 (réimpression 2006, l'Harmattan). 
idéologique, que la manifestation de sa supériorité intrinsèque, héritée des guerriers conquérants dont l'aristocratie continuait à se réclamer à la veille de la Révolution.

Avec l'apparition d'une forme administrative à la fin du Moyen Age pour exercer les fonctions régaliennes au nom du Roi, l'aristocratie finit par ne plus exercer elle-même directement les pouvoirs souverains, elle les délègue à des spécialistes méritants, et/ou fortunés, dont certains sont issus d'autres classes ${ }^{16}$. Ceci fait certes émerger de nouvelles classes spécifiquement liées au développement matériel et organisationnel de l'État mais, jusqu'à la fin de l'Ancien Régime, l'État sera considéré tout à fait explicitement par la noblesse française comme sa chose exclusive et c'est précisément contre ce monopole aristocratique qu'écrit Sieyès. C'est désormais à la Nation, qu'il assimile au Tiers État, de s'approprier le pouvoir politique, c'est-à-dire de contrôler l'État en son nom propre. Cette approche politique de la nation est inclusive par définition, elle s'oppose d'emblée à une vision ethno-nationale, laquelle est exclusive par définition. S'impose l'idée que le souverain n'est pas en haut mais en bas, la source du pouvoir est le peuple (ou la Nation, c'est tout un). L'État doit devenir une chose « publique » et ne plus demeurer le domaine d'une classe privilégiée, comme c'est le cas sous l'Ancien Régime. Il s'agit donc de construire un Etat pour l'ensemble des habitants du territoire. Les citoyens se constituent, plus ou moins brutalement, comme nation, laquelle devient alors l'organisation politique du peuple, et à terme, une fois le suffrage censitaire liquidé, le citoyen est pleinement fait cosouverain ${ }^{17}$. L'État peut alors devenir non pas l'agent des dominants mais du peuple dans son ensemble, même si la société demeure structurée en classes. C'est une contradiction profonde : l'égalité s'institue (progressivement) en droit, mais bien sûr pas en fait. Et la chose inouïe est que tous, par principe, paient l'impôt. Mais, les puissants n'ont jamais vraiment accepté ce nouvel état des choses car ils se pensent au-dessus des autres êtres humains, au-dessus des lois communes. Ils veulent faire leurs propres lois.

L'abolition des privilèges et le processus révolutionnaire conduit à rompre, avec succès, le lien organique et juridique entre l'État et l'aristocratie d'Ancien Régime. En revanche, le lien entre l'État et les classes dominantes demeure. Jusqu'à aujourd'hui, toute la contradiction de l'État moderne est là.

L'État est toujours un État de classe, mais quelque chose de nouveau a lieu. Avec le surgissement de la nation ${ }^{18}$, donc de la démocratie, qui intervient en France par le processus révolutionnaire, les classes dominantes ne peuvent plus revendiquer l'État comme étant « leur » chose exclusive. Pour contourner cette contradiction, elles imposent, au fil des luttes politiques, des formes de restrictions au vote selon la justification que la nation ne peut être arbitrairement représentée, chose classique autrefois, ou invoquent une forme de compétence politique légitimant implicitement leur place éminente dans l'Etat. La concentration du capital, propre aux moyens de communications, permet de justifier la confiscation du pouvoir au profit d'une supposée « expertocratie » ou « méritocratrie » ce qui n'abolit pas la conflictualité politique. Face à la réalité des rapports de classes, jusque dans l'Etat même, la nation impose une tension politique permanente car la nation englobe toute la société, elle est un au-delà des classes ${ }^{19}$. C'est un tout fantasmé pensent certains, mais il s'agit en réalité d'une fiction partagée dans le cadre de représentations communes et expérimentée collectivement, au premier rang desquelles se trouve l'égalité devant la loi. Elle

${ }^{16}$ Cf. Descimon, Robert, Guery Alain, Le Goff, Jacques, La longue durée de l'Etat, Seuil « Points », 2000 et Genet, Jean-Philippe, La genèse de l'Etat moderne, Puf, 2003.

17 Claude Nicolet, Histoire, Nation, République, Odile Jacob, 2000. Précisons que le terme souverain signifie « qui est au dessus de tout », il vient du latin supero, signifiant s'élever au-dessus, cf. Gaffiot, Félix, Dictionnaire latin français, (version V. M. Komarov) Gérard Gréco, (1934) 2016.

18 Delannoi définit la nation comme une « double forme » à la fois culturelle et politique « en partie disjoints, en partie superposés », cf. Delannoi, Gil, La nation contre le nationalisme, Puf, 2018, p. 34 et suiv.

${ }^{19}$ Cette contradiction de l'Etat, pris entre nation et structure de classe, n'est pas spécifique aux pays capitalistes avancés, elle se retrouve également sous des formes propres dans leurs anciennes colonies, voir notamment l'article de Rahmane Idrissa, « Des élites contre la nation : la Révolution africaine au Sahel », dans ce même dossier d'Actuel Marx. 
devient réalité et produit des effets tangibles. Comme tout fait humain, elle a une dimension immatérielle, morale disait-on autrefois. Cette représentation partagée a des effets bien réels et s'incarne dans des institutions qui appartiennent à notre matérialité ${ }^{20}$. Comme le souligne Maurice Godelier $^{21}$, si l'homme produit en société, il produit de la société pour vivre. Or, produire de la société, c'est inventer des fictions, des modes d'être collectif, bref, de la culture. La réalité politique, sociale et culturelle se double d'un imaginaire instituant qui vise à son auto-affirmation, donc à affirmer l'intérêt national qui se veut un intérêt dépassant les intérêts de classe. La chose estelle réellement possible? Sans doute pas, mais c'est assurément une manière euphémisée d'affirmer les intérêts du plus grand nombre face aux intérêts des classes possédantes minoritaires, lesquelles n'oseraient pas se prononcer directement contre la nation. La contradiction est toujours là : la nation a un potentiel émancipateur, démocratique, elle est un imaginaire instituant pour les classes dominées qui rêvent d'un monde sans classe, un monde d'égaux, mais les classes dominantes sont tout de même censées faire également partie intégrante de la nation malgré leur préférence pour le cosmopolitisme visible dès le XVIIIème siècle ${ }^{22}$. Désormais, l'enjeu pour les classes dominantes consiste à maintenir leur position de classe, lesquelles sont objectivement opposées à la nation, tout en ne se présentant pas contre mais avec la nation. Elles se disent favorables à la démocratie tout en invoquant sans cesse des contraintes extérieures de tous ordres, la normativité en provenance de l'Union européenne est exemplaire à cet égard. C'est la raison pour laquelle, dans le monde capitaliste contemporain, il est impossible pour les classes dominantes de revendiquer ou même de reconnaître la réalité de l'État de classe.

Les nations s'instituent à travers des régimes politiques et juridiques qui, d'une part, codifient les modalités de l'exercice des pouvoirs et, d'autre part, sont porteurs de l'imaginaire national. En France, la Nation prend la forme de la République. Dans ce pays, le premier régime démocratique fut la toute première des républiques. L'exemple des Jacobins a fasciné, y compris contre la France dans ses aventures impériales, car l'idéal de la nation moderne est profondément égalitaire et démocratique. Ainsi, la question nationale est à considérer du point de vue du mouvement qu'elle impulse dans l'histoire : issu du régime politique, la démocratie tend naturellement à investir l'économie. Avec l'échec de la deuxième République, s'impose alors l'idée qu'il faut quelque chose de plus pour réconcilier la république avec elle-même et viser l'égalité réelle : le socialisme. C'est ainsi que Jaurès y voyait le socialisme comme l'accomplissement même de la République ${ }^{23}$. On retrouve une perspective équivalente chez Gramsci ${ }^{24}$. Comme promesse instituante du dépassement des rapports de classe, la République est donc sociale par vocation. En attendant que cet horizon se réalise, elle œuvre non pas à l'exacerbation des rapports de classes mais à l'atténuation de leur violence.

${ }^{20}$ «L'imaginaire (mais non irréelle) communauté politique qui se met alors en place -celle disant l'égalité et la liberté des individus hors de tout présupposé relationnel-, est non pas d'abord le produit d'une conscience (individuelle ou collective), mais l'effet d'un mouvement objectif par lequel la société s'organise politiquement », cf. Artous, Antoine, Marx, l'Etat moderne et la sociologie de l'Etat, L'Homme et la Société, 136-7, sept, 2000 : 111-126.

${ }^{21}$ Maurice Godelier, L'idéel et le matériel, Fayard, 1984.

${ }^{22}$ Voici ce qu'affirme Rousseau, dans un chapitre introductif de la première version du Contrat Social: «Par où l'on voit ce qu'il faut penser de ces prétendus cosmopolites, qui, justifiant leur amour pour la patrie par leur amour pour le genre humain, se vantent d'aimer tout le monde pour avoir droit de n'aimer personne ", cité par Ghorbel, Hichem, L'idée de guerre chez Rousseau. Volume 2 - Paix intérieure et politique étrangère, L'Harmattan, 2010, p. 100. Pour une approche critique, en terme de rapports de classe, du cosmopolitisme contemporain au Royaume-Uni et aux Etats-Unis, cf. Goodhart, David, The road to somewhere, Hurst \& co., 2017 et Bageant, Joe, Deer Hunting with Jesus, Crown Business, 2007.

${ }^{23}$ Sur ces questions, voir notamment l'article de Lucien Calvié, « Nations, nationalismes, marxismes, révolutions et républiques : les cas allemand et slave », dans ce même dossier d'Actuel Marx.

${ }^{24}$ Sur cette question, voir l'article de Jean-Numa Ducange « Faut-il défendre la nation ? Marx, les marxistes et la question nationale des origines à nos jours », dans ce même dossier d'Actuel Marx. 
Ce n'est pas un hasard si ceux qui désiraient un ordre libéral sans démocratie étaient ostensiblement anti-républicains. Au contraire, le moment de la Commune ${ }^{25}$, on l'oublie trop souvent, illustre à l'extrême la fusion de la gauche avec le patriotisme. Une politique anti-républicaine ou antinationale incite à la révolte, à la guerre civile, elle se présente donc comme une politique des classes dominantes pour les classes dominantes, c'est-à-dire contre la nation. La République, forme concrète et particulière de la démocratie, est donc nécessairement imparfaite puisque ce n'est qu'une réalisation. Elle repose sur l'État de droit et l'égalité devant la loi. Bien que ces deux éléments ne demeurent pas toujours inviolés, ils servent de boussole, de bassin d'attraction, bref ils sont instituants. Les expériences socialistes sans exigence républicaine ont engendré des régimes politiques peu respectueux de la personne. Pour ceux-ci, l'égalité formelle n'est qu'un embarras et ils en viennent à recréer une classe dirigeante incarnant l'État au nom du meilleur des mondes. Le « socialisme réel » a généré un monde hybride : d'un côté, on retrouve des éléments de « l'Etat médiéval », où la distinction entre la classe dominante et l'Etat est peu claire, et, d'un autre côté, un Etat social est mis en place.

L'emploi des termes « Etat de classe » ne signifie pas ici un Etat dont serait propriétaire une classe, comme ce fut le cas dans le passé. Le compromis propre aux démocraties parlementaires implique que la puissance de l'Etat ne soit pas formellement au service exclusif d'une classe, ou d'un groupe de classes, pour achever ses objectifs propres. L'Etat de classe, étant également et de manière contradictoire Etat-nation, n'est donc pas un Etat dont la classe dominante accéderait ainsi à la souveraineté et serait en mesure de décider de ce qui est exceptionnel pour s'affranchir des règles établies, comme c'est le cas avec le fascisme ${ }^{26}$. De même, dans ce cadre, les classes dominées ne peuvent faire de l'Etat un instrument au service de la révolution sociale. L'Etat est contradictoire, il est simultanément « nation » et de « de classe ». Dès lors, il devient l'espace où s'exprime la conflictualité sociale, étant donné que l'inégal accès aux ressources économiques est une donnée essentielle des rapports de forces politiques. Ce fait n'implique pas que la coalition des classes subordonnées, qui ne sont pas en état de dominer économiquement, ne puisse influencer en profondeur le jeu politique. C'est précisément pour pallier cette influence sociale et politique des classes dominées que les fascistes ont liquidé les institutions démocratiques-parlementaires et envoyé, au mieux, les opposants dans les camps. Le fascisme révèle que la démocratie n'est pas une superstructure du capitalisme ${ }^{27}$.

À ce jour, les nations organisées politiquement en démocraties ont été le cadre d'une solidarité et d'une redistribution encore inégalés dans l'histoire. L'autre bifurcation historique, c'est en effet l'Etat social et son impôt progressif. Il germe en Europe occidentale sur les ruines de la Commune de Paris. Il prendra son essor d'abord en Allemagne dès les années 1880, et s'imposera sous la menace du stalinisme, à la faveur de la crise de 1929, puis de la Seconde guerre mondiale. Depuis le début, les classes dominantes le subissent, elles résistent : c'est le sens même du néolibéralisme. En dépit des idéologies, le keynésianisme et la Sécurité sociale (en France) donnent toutefois la forme concrète d'un début de république sociale. La droite et la gauche se succèdent au pouvoir dans une république sociale, certes inachevée mais en progrès constant (jusqu'en 1983).

\footnotetext{
${ }^{25}$ Etrangement, la Commune est aujourd'hui vantée par une fraction de la gauche qui rejette l'idée de nation. Le parti des Le Pen fait la même chose avec le mythe de Jeanne d'Arc. Or, celui-ci fut porté par les républicains au XIXème siècle, c'est-à-dire, alors, par la gauche : «Il s'agissait de montrer que, dès l'origine, le peuple avait aimé la France, alors que les nobles étaient des traîtres », cf. Beaune, Colette, Jeanne d'Arc, ancienne et nouvelles légendes, pp. 3744, dans Weill-Parot, Nicolas et Sales, Véronique Le vrai visage du Moyen-Age-au-delà des idées reçues, Vendémiaire, 2017. On retrouve cette même conviction chez le communard Gustave Lefrançais, dans ses inoubliables Souvenirs d'un révolutionnaire - De juin 1848 à la Commune, préface de Daniel Bensaïd, La Fabrique 2013.

${ }^{26}$ Cf Carl Schmitt, La dictature, Pocket, (1921) 2015.

${ }^{27}$ Cf. Maucourant, Jérôme, La démocratie est-elle une superstructure du capitalisme ? Un regard de Karl Polanyi sur le marxisme, Revue du MAUSS permanente, 13 mars 2010 [en ligne]. http://www.journaldumauss.net/./?Lademocratie-est-elle-une
} 
Par l'État social mis en place au 20ème siècle et par les politiques de plein emploi, la solidarité nationale a permis d'atteindre un niveau de confort matériel pour l'ensemble de la population, inimaginable encore il y a cent ans. Jamais, au cours de l'expérience humaine, notre espèce n'avait atteint une telle longévité en bonne santé pour une fraction aussi large de la société. La mondialisation et l'effritement des souverainetés nationales conduisent à l'érosion des protections sociales et un relâchement des solidarités nationales, si bien que des reculs dans l'espérance de vie des catégories les plus fragiles de la population s'observent depuis la crise de 2008-9. Loin de se réduire au nationalisme, au chauvinisme ou au repli sur soi, la nation a au contraire montré qu'elle peut être le lieu du progrès social et de l'émancipation. Pour l'instant, aucune autre entité n'a su faire mieux, ni même aussi bien. La nation est la base de l'internationalisme compris non pas comme un mondialisme, lequel sert d'abord les classes dominantes contre leurs nations, mais comme la coopération de peuples libres, c'est-à-dire maîtres de leurs destins par des processus démocratiques. La nation est comme un patrimoine que chaque génération améliore ou dilapide : de ce passé faut-il faire table rase au nom d'une Europe sans peuple ou d'un internationalisme sans nations ?

\section{Un monde post-national et post-souverain ?}

La tendance de l'État est de conserver la structure de classe. Malgré cette limite, le champ des possibles demeure largement indéterminé, il dépend des luttes et des compromis de classe qui se nouent et se dénouent. Surtout, l'État-nation a déjà montré qu'il est en mesure de subvertir l'ordre de classe par des politiques de plein emploi et le développement de l'État social, lequel tend à soustraire la reproduction de la force de travail de la loi du marché. Pour faire court, notre État moderne est un État de classe, certes, mais avec protection sociale. Devenu une force relativement autonome, il est assiégé par les classes dominantes qui font tout leur possible pour en garder le contrôle exclusif par leurs lobbies et divers groupes de pression externes et internes. En raison du caractère devenu national, donc démocratique, des États modernes, la victoire des classes dominantes ne peut être totale : d'une manière ou d'une autre, les classes dominées exigeront des protections contre les effets délétères des choix politiques et économiques imposés par les classes possédantes.

La constitutionnalisation de la politique économique contre les nations revient, pour les gouvernements, à abdiquer certaines de leurs prérogatives. Cette abdication n'est pas le fruit d'une soumission à la nécessité, thèse dominante sur la mondialisation faisant d'elle une force irrésistible s'imposant de l'extérieur aux États-Nations. Si ces dessaisissements partiels de souveraineté ont pu avoir lieu, c'est en raison de la conversion des « élites » politiques nationales au credo des groupes socio-économiques dominants. Les gouvernants se sont auto-imposés des contraintes externes afin de réduire les coûts politiques de leurs choix économiques, en les imputant à des éléments tiers comme « les institutions européennes », « les marchés », « la concurrence mondiale » etc. Les délégations de souveraineté furent effectuées dans le but de mieux domestiquer les classes subalternes. Les gouvernements ont pu imposer plus efficacement des choix de politique économique et de « réformes » souhaités par les élites aux classes dominées, au nom de la « flexibilité », « l'impératif européen », etc. On pourrait affirmer que l'UEM est un projet international mais est-ce si sûr? Il contribue à désarticuler les nations et cherche à les dépasser au profit d'une « gouvernance européenne » qui n'est que le « gouvernement représentatif » des intérêts établis du capitalisme européen.

C'est pourquoi il faut qualifier d'idéologiques les affirmations selon lesquelles un ensemble de forces techno-marchandes, tout aussi irrésistibles et que spontanées -quasi-naturelles-, auraient fait reculer l'État malgré lui. En réalité, le capital n'est pas moins indépendant de l'État aujourd'hui qu'il ne l'était durant la période d'après-guerre. Désormais, les crises se multiplient, ce qui nécessite d'autant plus qu'il intervienne directement en sa faveur, par diverses formes de plans de sauvetage. L'État ne s'est pas effacé pour faire supposément place au marché, ses modalités d'action ont été redéfinies pour faire primer le règne de la marchandise. Tout aussi erronée est l'idée, très répandue, 
que la souveraineté des États-Nations aurait été érodée et réduite à néant par la globalisation financière. L' « internationalisation » économique et financière aurait tellement affaibli la capacité des États à contrôler leurs propres économies, qu'ils n'auraient plus d'autre choix que de cesser d'intervenir au niveau national. En compensation, la seule issue consisterait à s'en remettre à une autorité supranationale, voire mondiale, dont on formule le vœu qu'elle serait disposée à agir de manière plus favorable à l'endroit des gens ordinaires. Le monde serait devenu post-national et postsouverain.

Déjà, au 19ème siècle, avait émergé l'idée que la force du capital, sans cesse en recherche de nouveaux marchés et réorganisant continuellement la production, vient contraindre l'autonomie des États-nations. Pourtant, aujourd'hui encore le monde demeure composé de zones économiques nationales qui sont loin d'être intégrées les unes aux autres. C'est également vrai au sein de la zone Euro : après vingt ans de monnaie unique, il n'y a pas eu de convergence réelle et depuis la crise financière de 2008, puis celle de l'Euro en 2012 on observe plutôt le contraire. Dans quelle mesure, faudrait-il continuer à donner crédit au poncif selon lequel tout aurait changé à l'ère de la mondialisation au point de rendre caduque le rôle des États-nations ? Le recul de l'État-nation, mais pas de l'État tout court (c'est-à-dire l'État de classe), c'est surtout l'avancée du capital.

Les grandes entreprises, qui opèrent dans plusieurs pays, pour tirer avantage des différences de prix du travail d'une zone à l'autre, ont besoin des États pour faire prévaloir leurs droits au niveau national et international et elles sont utilisées par les États dominants pour asseoir leur pouvoir dans la hiérarchie du pouvoir international. Les grandes entreprises ont besoin de l'État pour créer les conditions de leur profitabilité et compenser les effets des crises tant au niveau national qu'international. La libéralisation du commerce international, la dérégulation, la privatisation des grandes entreprises publiques etc., ne sont pas le résultat de forces techno-économiques implacables mais bel et bien de changements réglementaires et institutionnels opérés par les gouvernements. Il serait erroné de se représenter les États et les gouvernements comme des victimes de la mondialisation dans la mesure où, lorsque des grandes entreprises semblent s'être autonomisées de leur pays d'origine, elles n'ont pu le faire que parce que les gouvernements composant les États du centre ont mis en place les structures institutionnelles qui rendent la mondialisation possible. Le libre échange promu par les classes dominantes des pays du centre a conduit à délocaliser massivement dans les pays à très bas salaires, faisant émerger pour les grandes entreprises internationales une gigantesque armée de réserve industrielle au niveau mondial. Non seulement les travailleurs employés dans les pays-ateliers, comme la Chine, se trouvent alors extraordinairement exploités, tant leur offre de travail est excédentaire, mais les travailleurs du centre sont également mis davantage sous pression, car subissant à distance cette concurrence, notamment par la menace de délocalisation. Le chantage aux emplois aurait-il été possible sans la mise en place, par les classes dominantes nationales, des dispositifs institutionnels qui font la mondialisation? Ces règles ont été fabriquées de manière à permettre à ces mêmes classes dominantes de capter un maximum de ressources, et de s'approprier une fraction très élevée -pour ne pas dire la totalité- des gains de la croissance.

Aujourd'hui comme il y a 100 ans, avant le new deal, la logique de la globalisation financière est celle du capital, et les gouvernements épousent plus ou moins volontiers cette logique. Elle n'a rien de naturel ni de technique, il s'agit d'une logique sociale. Les souverainetés nationales peuvent, si ce n'est y mettre fin, du moins y opposer des limites plus étroites. Certes, le champ des possibles s'étend avec l'essor de la technique, mais il revient toujours au législateur de tracer la limite entre le licite et l'illicite. Ainsi, les gouvernements facilitent plus ou moins la logique du capital selon les compromis politiques, dont une des dimensions est d'être des compromis de classe, qui sont temporairement forgés au sein de chaque nation. Comme le soulignent Bryan, Rafferty et Wigan ${ }^{28}$,

${ }^{28}$ Cf. Bryan, Dick, Rafferty, Michael and Wigan, Duncan, From time-space compression to spatial spreads, situating nationality in global financial liquidity, in Money and Finance after the Crisis: a Critical Thinking for Uncertain Times, edited by B. Christophers, A. Leyshon and G. Mann, Wiley \& Sons, 2017: 43-67; et Bryan, Dick et Rafferty, 
même la finance la plus éthérée, la plus abstraite et volatile que représentent les produits dérivés, dont les facultés vont jusqu'à modifier l'appréciation de l'espace et du temps par le capital, a malgré tout besoin de se matérialiser à un moment ou à un autre dans un espace national précis, donc dans un espace juridictionnel spécifique. Ceci au moins pour deux raisons : les contrats financiers relèvent toujours d'une juridiction donnée, qui apporte une grammaire pour écrire les contrats et un système de sanctions en cas de contentieux. Même si les produits dérivés semblent parfois au-dessus des territoires et hors du temps ordinaire, ils finissent par avoir besoin de se dénouer en un lieu et un moment précis afin que soient soldés les pertes et les profits entre les contractants. Voilà pourquoi, dans les nouveaux traités signés au cours des dernières décennies, le capital tente d'imposer directement son propre droit, ses propres procédures et même ses propres tribunaux. La bataille contre les souverainetés nationale ${ }^{29}$ est une bataille politique et culturelle engagée de longue date par le capital.

\section{Quelle politique économique aujourd'hui ?}

Ce qui se joue dans de nombreux pays depuis Thatcher, et s'accélère après 2008-9, c'est le retour à un État de classe contre la nation, mais contrairement au début du 20ème siècle, c'est un État de classe avec protection sociale. Les décennies de plein emploi qui ont suivi l'après-guerre ont fini, durant les années 1970, par poser une menace à la reproduction même des rapports de production capitaliste, ce qui mettait à l'ordre du jour le dépassement de ce système économique. A lui seul, le keynésianisme n'a pas permis malgré le plein emploi de répondre aux attentes des salariés qui aspiraient à de meilleurs salaires, certes, mais aussi à une autre organisation du travail, à de plus faibles inégalités et, surtout, à un contrôle social de l'accumulation du capital. Autrement dit, la socialisation (d'une fraction) de la production se devait de venir compléter la (fraction de la) consommation socialisée par l'avènement de l'État social.

Les voies du progrès social et de l'émancipation passent par la reprise en main de leurs souverainetés par les États-Nations. C'est à ce niveau que peuvent encore se mobiliser et s'exprimer les intérêts des classes qui paient le plus lourd tribut face aux politiques menées depuis quatre décennies.

Alors que l'État est en train de redevenir la chose exclusive des classes dominantes, la dépense et la socialisation de l'investissement peuvent au contraire faire en sorte qu'il soit à nouveau l'affaire de tous. Les États-Nations sont loin de disparaître et c'est pourquoi, au lieu d'invoquer un internationalisme sans nation, improbable «alter-mondialisme », il serait sage de voir à nouveau la nation comme lieu possible de dépassement des rapports de classe. La République n'est pas un état figé du développement politique, c'est plutôt un processus vers davantage de démocratie et d'égalité sociale, c'est-à-dire un monde orienté par les besoins et l'émancipation de tous. Quel autre projet sérieux s'offre à nous ? Un danger existe et n'est jamais à exclure, en cas de crise générale et durable : c'est bien sûr celui du retour de la nation identitaire et conservatrice des hiérarchies sociales, car on n'enlèvera jamais au groupes humains le désir de s'enraciner (sauf dans l'élite mondialiste), bref de s'appartenir concrètement. Les coups portés par le capital transnational et les dérives idéologiques multiples (de « droite » comme de « gauche ») qui lui sont associées, auront une part de responsabilité terrible dans ce cas. Mais, pour ne pas en venir à ce stade-là, le médiéviste marxiste Guy Bois nous avertissait, il y a près de vingt ans, à propos de la

Michael, Capitalism with derivatives. A Political Economy of Financial Derivatives, Capital and Class, Palgrave Macmillan, 2006.

${ }^{29}$ Polanyi, un contemporain de cette période, rappelle à ce propos quelques décennies après : « Une paix de Cent ans avait bâti une insurmontable montagne d'illusions qui cachait les faits. Ceux qui ont écrit pendant cette période ont excellé dans le manque de réalisme. A. Toynbee estimait que l'Etat-nation était un préjugé étroit, Ludwig von Mises que la souveraineté était une illusion ridicule. Norman Angell que la guerre était un faux calcul d'affaire. La conscience que les problèmes politiques sont essentiels était tombée plus bas que jamais ». Cf. Polanyi, Karl, $L a$ Grande Transformation, Gallimard, 1983, p. 251. 
mondialisation comme « nouvelle servitude » : c'est sur « le terrain idéologique que la partie se jouera $\rangle^{30}$. Avec la crise sanitaire, cette partie peut prendre une ampleur inédite.

${ }^{30}$ Guy Bois, Une nouvelle servitude - essai sur la mondialisation, de Guibert, 2003, p. 175. 